\title{
Structured and Reduced Dimension Explicit Linear Quadratic Regulators for Systems with Constraints
}

\author{
Tor A. Johansen \\ Department of Engineering Cybernetics, Norwegian University of Science and Technology, \\ 7491 Trondheim, Norway. Tor.Arne.Johansen@itk.ntnu.no
}

\begin{abstract}
It is studied how system structure can be utilized to derive reduced dimension multi-parametric quadratic programs that lead to sub-optimal explicit piecewise linear feedback solutions to the state and input constrained LQR problem. This results in a controller of lower complexity and associated computational advantages in the online implementation. At heart of the methods are state space projections using the singular value decomposition.
\end{abstract}

\section{Introduction}

In this work we consider constrained linear quadratic regulators (LQR) $[1,2]$. Recently, approaches to the design of explicit solutions in terms of a piecewise linear (PWL) state feedback have been developed $[3,4,5,6]$. In particular, numerical algorithms for multi-parametric quadratic programming (mp-QP) has opened for the efficient and exact design of such PWL state feedbacks defined on polyhedral partitions of the state space. However, the complexity of the polyhedral partition often increases rapidly with the dimension of the state vector, and the number of constraints. This has led to several approximate algorithms for solving mp-QP problems being investigated, [7,8], with significant reduction in complexity. Moreover, it has led to the investigation of efficient implementation of piecewise linear function evaluation $[9,10]$ as well as input trajectory parameterization $[10]$ and restrictions on the active constraint switching $[11,12]$ in order to reduce the complexity.

In the present work we take a different approach to complexity reduction, which can be used in combination with any of the approaches mentioned above. It is based on the idea that in systems with significant structure (such as cascaded or weakly interconnected subsystems) and with constraints only on a relatively small number of state variables, one may be able to exploit this structure in order to define an approximate $\mathrm{mp}-\mathrm{QP}$ problem on a sub-space of the state space. This results in a PWL state feedback defined on a lower-dimensional space, combined with a full linear state feedback. The benefit of this is that the mp-QP of reduced dimension requires less computer processing and memory, both offline and online.

\section{Explicit Constrained Linear Quadratic Regulator}

Formulating the constrained LQR problem as an mp-QP is briefly described below, see [4] for further details. Consider the linear system

$$
x(t+1)=A x(t)+B u(t)
$$

where $x(t) \in \mathbb{R}^{n}$ is the state variable, and $u(t) \in \mathbb{R}^{m}$ is the input variable. For the current $x(t)$, the constrained LQR solves the optimization problem

$$
V^{*}(x(t))=\min _{U \triangleq\left\{u_{t}, \ldots, u_{t+N-1}\right\}} J(U, x(t))
$$

subject to (for $k=0,1, \ldots, k-1$ )

$$
\begin{aligned}
& y_{\min } \leq y_{t+k+1 \mid t} \leq y_{\max } \\
& u_{\min } \leq u_{t+k} \leq u_{\max } \\
& x_{t+k+1 \mid t}=A x_{t+k \mid t}+B u_{t+k} \\
& y_{t+k+1 \mid t}=C x_{t+k+1 \mid t}
\end{aligned}
$$

with $x_{t \mid t}=x(t)$ and the cost function given by

$$
\begin{gathered}
J(U, x(t))=\sum_{k=0}^{N-1}\left(x_{t+k \mid t}^{T} Q x_{t+k \mid t}+u_{t+k}^{T} R u_{t+k}\right) \\
+x_{t+N \mid t}^{T} P x_{t+N \mid t}
\end{gathered}
$$

We assume symmetric $R \succ 0$ (positive definite), $Q \succeq 0$ (positive semi-definite), $(A, B)$ is controllable and $(A, \sqrt{Q})$ is observable. The symmetric final cost matrix $P \succ 0$ is taken as the solution of the algebraic Riccati equation. With the assumption that no constraints are active for $k \geq N$ this corresponds to an infinite horizon LQ criterion $[1, \overline{2}]$. With proper definitions of the matrices $Y, H, F, G, W$ and $E$, see $[11,4]$, this and related problems can be reformulated as follows: Minimize with respect to $U$

$$
\begin{aligned}
J(U, x)= & \frac{1}{2} x^{T} Y x+\frac{1}{2} U^{T} H U+x^{T} F U \\
\text { subject to } & G U \leq W+E x
\end{aligned}
$$

It is shown in [4] that $H \succ 0$ due to $R \succ 0$, such that this problem is strictly convex. Completing squares in (5)-(6), the dependence on $x$ is moved from the cost to the constraints, such that the problem is equivalent to the following:

$$
\begin{aligned}
& V_{z}^{*}(x)=\min _{z} \frac{1}{2} z^{T} H z \\
& \text { subject to } \quad G z \leq W+S x
\end{aligned}
$$

where $z=U+H^{-1} F^{T} x$ and $S=E+G H^{-1} F^{T}$. We let the unconstrained LQ optimal control be denoted $U_{L Q}(t)=$ $-K_{L Q} x(t)$ where $K_{L Q}=H^{-1} F^{T}$ is an extended LQ gain matrix. We let the $m$ first rows of $U_{L Q}(t)$ be denoted $u_{L Q}(t)$, and the corresponding $m$ first rows of $K_{L Q}$ be denoted $k_{L Q}$, the usual LQ gain matrix. Eqs. (7)-(8) defines a strictly convex mp-QP in $z$ parameterized by $x \in X$, where $X$ is a polyhedral set. This mp-QP can be solved using the algorithms in $[4,6]$, which give the solution $z^{*}(x)$ as an explicit function of $x \in X$ with the following properties [4]:

Theorem 1 Consider the $m p-Q P(7)-(8)$ with $H \succ 0$. The solution $z^{*}(x)$ (and $\left.U^{*}(x)=z^{*}(x)-H^{-1} F^{T} x\right)$ is a continuous $P W L$ function of $x$, and $V_{z}^{*}(x)$ is a convex and continuous piecewise quadratic function. 


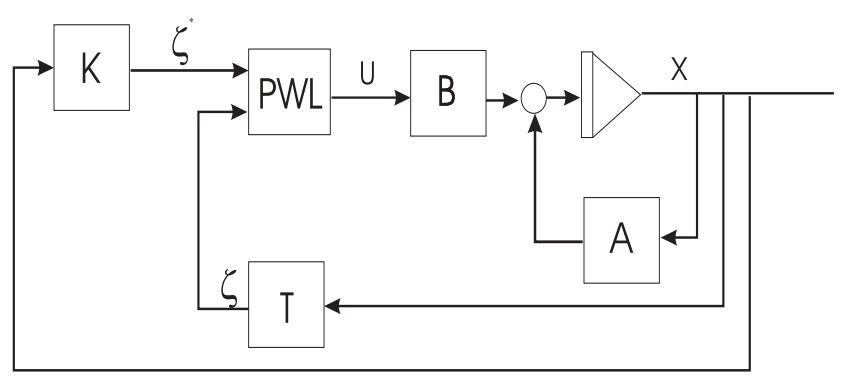

Figure 1: Feedback structure I.

The concept of active constraints is instrumental to characterizing the PWL solution. An inequality constraint is said to be active for some $x$ if it holds with equality at the optimum. An explicit representation of the optimal PWL state feedback is given as follows [4]:

Theorem 2 Consider the mp- $Q P$ (7)-(8) with $H \succ 0$, and an arbitrary fixed set of active constraints, where the submatrices $\tilde{G}, \tilde{W}$ and $\tilde{S}$ contain the corresponding rows of $G$, $W$ and $S$. If the rows of $\tilde{G}$ are linearly independent, the optimal solution and associated Lagrange multipliers are given by the affine functions

$$
\begin{aligned}
& z_{0}^{*}(x)=H^{-1} \tilde{G}^{T}\left(\tilde{G} H^{-1} \tilde{G}^{T}\right)^{-1}(\tilde{W}+\tilde{S} x) \\
& \tilde{\lambda}_{0}(x)=-\left(\tilde{G} H^{-1} \tilde{G}^{T}\right)^{-1}(\tilde{W}+\tilde{S} x)
\end{aligned}
$$

Moreover, the critical region $C R_{0} \subseteq \mathbb{R}^{n}$ where this solution is optimal is given by the polyhedron

$$
\begin{aligned}
G H^{-1} \tilde{G}^{T}\left(\tilde{G} H^{-1} \tilde{G}^{T}\right)^{-1}(\tilde{W}+\tilde{S} x) & \leq W+S x \\
-\left(\tilde{G} H^{-1} \tilde{G}^{T}\right)^{-1}(\tilde{W}+\tilde{S} x) & \geq 0
\end{aligned}
$$

The complexity of solving the mp-QP and implementing the resulting PWL state feedback increases rapidly with the number of constraints and the dimension of the state space. In this work we suggest some methods for reducing complexity, where we essentially aim to replace the linear terms $E x$ and $F^{T} x$ in (5)-(6) or $S x$ in (8) with approximate linear mappings defined on a sub-space of the state space. This leads to new mp-QPs defined on a lower-dimensional parameter space, with associated computational advantages.

\section{Feedback structure I}

Consider the feedback structure in Figure 1. It contains an inner PWL feedback loop, to be designed by solving an mp$\mathrm{QP}$, and a linear feedback in the outer loop, to be designed to achieve local LQ optimality. The idea is that the inner PWL loop relies on feedback from a reduced state $\zeta=T x$, where the projection matrix $T \in \mathbb{R}^{p \times n}$, with $2 p<n$, is chosen such that it contains the necessary information to guarantee closeto-optimal control of $\zeta$ to its specified setpoint $\zeta^{*}$, while fulfilling all constraints on $x$ and $u$. This amounts to solving an mp-QP with $2 p$ parameters.

Lemma 1 The constraints (6) are equivalent to

$$
G U \leq W+E_{0} \zeta
$$

where $\zeta=T x$ is defined by the projection matrix $T=V_{0}^{T}$, and $E_{0}=U_{0} \Sigma_{0}$, where $U_{0}, V_{0}, \Sigma_{0}$ are the sub-matrices of the singular value decomposition $E=U \Sigma V^{T}$ corresponding to non-zero singular values.

Proof. $E x=U \Sigma V^{T} x=U_{0} \Sigma_{0} V_{0}^{T} x=E_{0} T x=E_{0} \zeta$, cf. [13].

Theorem 3 The row rank of the observability matrix $W_{o}=$ $\left(C^{T},(C A)^{T}, \ldots,\left(C A^{n-1}\right)^{T}\right)^{T}$ of the system $(A, C)$ is an upper bound on the number of non-zero singular values of $E$, i.e. $p=\operatorname{dim}(\zeta)=\operatorname{rank}(E) \leq \operatorname{rank}\left(W_{o}\right)$.

Proof. For input constraints, the corresponding rows of $E$ are zero. For a generic output constraint $y_{\min } \leq y(t+k) \leq$ $y_{\max }$ the corresponding block of $E$ is $C A^{k}$, see e.g. [11]. Hence, $E$ can be written

$$
E=\left(\begin{array}{c}
0_{2 N m \times n} \\
W_{N} \\
-W_{N}
\end{array}\right)
$$

where the first block corresponds to input constraints and the two last blocks corresponds to the output constraints with $W_{N}$ being the Krylov matrix

$$
W_{N}=\left(C^{T},(C A)^{T},\left(C A^{2}\right)^{T}, \ldots,\left(C A^{N}\right)^{T}\right)^{T}
$$

For $N \geq n$, the row rank of $W_{N}$ equals the row rank of $W_{o}$ due to Cayley-Hamiltons theorem [14]. The row rank of $E$ equals the row rank of $W_{N}$, from(14), and the result follows by Lemma 1 .

The above results suggest that for the purpose of fulfilling the constraints it is sufficient to use information only about those modes of the system that are observable from the output $y=C x$, which are the constrained modes. Obviously, the reformulation (13) makes sense only if it is possible to find a projection matrix with $p<n / 2$, since otherwise there will be no reduction in the dimension of the parameter space. The approach is also meaningless if there are only input constraints or if $p<m$. On the other hand, the idea is expected to be useful when the system possesses significant structure, such as a cascade where all constrained states are "close to the inputs" in the sense that there are relatively few integrators between the inputs and the constrained states. The suggested feedback structure may also be useful in an approximate setting. In this case $p$ will equal the number of singular values of $E$ that are significantly larger than zero. In order to design the feedback laws, we introduce the similarity transform

$$
\left(\begin{array}{l}
\zeta \\
\varrho
\end{array}\right)=V^{T} x
$$

where the vector $\zeta$ contains the $p$ modes that are observable through $y$ from the constrained states, and $\varrho$ the $n-p$ modes that are not. Hence, the following projections hold: $\zeta=$ $V_{0}^{T} x, \varrho=V_{1}^{T} x$ with $V_{0} \in \mathbb{R}^{n \times p}$ and $V_{1} \in \mathbb{R}^{n \times(n-p)}$. Since $V$ is orthogonal, the inverse transform is given by $x=V_{0} \zeta+$ $V_{1} \varrho$. We define the following projected matrices $F_{0}=V_{0}^{T} F$, $F_{1}=V_{1}^{T} F, Y_{00}=V_{0}^{T} Y V_{0}, Y_{01}=Y_{10}^{T}=V_{0}^{T} Y V_{1}$, and $Y_{11}=V_{1}^{T} Y V_{1}$. We are then in position to reformulate the 
cost function (5) into the form

$$
\begin{aligned}
J(U, \zeta(t), \varrho(t))= & \frac{1}{2} U^{T} H U+\left(\zeta(t)-\zeta^{*}\right)^{T} F_{0} U \\
& +\frac{1}{2}\left(\zeta(t)-\zeta^{*}\right)^{T} Y_{00}\left(\zeta(t)-\zeta^{*}\right) \\
& +\zeta^{* T} F_{0} U+\varrho^{T}(t) F_{1} U \\
& +\varrho^{T}(t) Y_{10} \zeta(t)+\frac{1}{2} \varrho^{T}(t) Y_{11} \varrho(t) \\
& -\frac{1}{2} \zeta^{* T} Y_{00} \zeta^{*}+\zeta^{T}(t) Y_{00} \zeta^{*}
\end{aligned}
$$

where we have introduced the new variable $\zeta^{*}$, whose value does not influence the value of $J$. We now develop a suboptimal strategy by separating the three first terms:

$$
\begin{aligned}
J_{0}\left(U, \zeta(t), \zeta^{*}\right)= & \frac{1}{2} U^{T} H U+\left(\zeta(t)-\zeta^{*}\right)^{T} F_{0} U \\
& +\frac{1}{2}\left(\zeta(t)-\zeta^{*}\right)^{T} Y_{00}\left(\zeta(t)-\zeta^{*}\right)
\end{aligned}
$$

subject to

$$
G U \leq W+E_{0} \zeta(t)
$$

Assuming $2 p<n$, (18)-(19) define a reduced-dimension mpQP on a $2 p$-dimensional sub-space of the state space, and from the results above it is guaranteed that for any $\zeta^{*}$ the original constraints (6) are fulfilled. When solving the mpQP (18) - (19) the set $\Upsilon \times \Upsilon^{*}$ of possible $\left(\zeta, \zeta^{*}\right)$ must be specified. Polyhedral $\Upsilon$ and $\Upsilon^{*}$ can be specified by projections of the polyhedral set $X$ :

$$
\begin{aligned}
\Upsilon & =\{\zeta \mid \zeta=T x, x \in X\} \\
\Upsilon^{*} & =\left\{\zeta^{*} \mid \zeta^{*}=K x, x \in X\right\}
\end{aligned}
$$

Let the PWL solution to (18)-(19) be denoted $U_{0}^{*}\left(\zeta, \zeta^{*}\right)$ and the first $m$ elements of this vector be denoted $u_{0}^{*}\left(\zeta, \zeta^{*}\right)$. The control is then given by the receding horizon policy

$$
u^{*}(t)=u_{0}^{*}\left(\zeta(t), \zeta^{*}(t)\right)
$$

The variable $\zeta^{*}$ is viewed as a reference signal to the inner loop, as shown in Figure 1. Since the constraints are guaranteed to be fulfilled with the PWL inner feedback loop described above, we restrict our attention to a (sub-optimal) linear outer loop that determines $\zeta^{*}=K x$. Let the gain matrix of the reduced-dimension unconstrained LQ design be denoted $k_{0} \in \mathbb{R}^{m \times p}$ and given by the $m$ first rows of the matrix $K_{0}=H^{-1} F_{0}^{T}$. Hence, $u=-k_{0}\left(\zeta-\zeta^{*}\right)$ coincides with the solution $u_{0}^{*}\left(\zeta, \zeta^{*}\right)$ of (18) - (19) in a neighborhood of the origin. Local LQ optimality follows if $K \in \mathbb{R}^{p \times n}$ is appropriately chosen:

Theorem 4 Suppose $p \geq m$, rank $\left(k_{0}\right)=m$, $y_{\min }<0$, $y_{\max }>0, u_{\min }<0, u_{\max }>0$. Then there exists a gain matrix $K$ that solves the system of linear equations

$$
k_{0} K=k_{0} T-k_{L Q}
$$

and the system (1) in closed loop with the control (22) and $\zeta^{*}(t)=K x(t)$ is locally (unconstrained) LQ optimal, with respect to $(4)$.
Proof. Notice that (23) defines $m n$ equations with $p n$ unknowns (recall $p \geq m$ )

$$
\left(\begin{array}{cccc}
k_{0} & 0 & & 0 \\
0 & k_{0} & & 0 \\
& & \ddots & \\
0 & 0 & & k_{0}
\end{array}\right)\left(\begin{array}{c}
K^{1} \\
K^{2} \\
\vdots \\
K^{n}
\end{array}\right)=\left(\begin{array}{c}
\left(k_{0} T-k_{L Q}\right)^{1} \\
\left(k_{0} T-k_{L Q}\right)^{2} \\
\vdots \\
\left(k_{0} T-k_{L Q}\right)^{n}
\end{array}\right)
$$

The superscript index denotes the corresponding column of a matrix. Due to $\operatorname{rank}\left(k_{0}\right)=m$ the matrix to the left has full row rank, and there exists a $K$ solving (24). Since $y_{\min }<0$, $y_{\max }>0, u_{\min }<0, u_{\max }>0$, it is clear that there exists a neighborhood of the origin where the optimal control $u^{*}(t)$ has no active constraints [4]. In this set, the dynamics of the inner feedback loop is given by

$$
x(t+1)=\left(A-B k_{0} T\right) x(t)+B k_{0} \zeta^{*}(t)
$$

In closed loop with the outer feedback loop $\zeta^{*}=K x$ this leads to

$$
\begin{aligned}
x(t+1) & =\left(A-B\left(k_{0} T-k_{0} K\right)\right) x(t) \\
& =\left(A-B k_{L Q}\right) x(t)
\end{aligned}
$$

and the result follows due to LQ optimality of (26).

If $p=m$ the system of linear equations (24) has a unique solution, while there are several solutions for $p>m$. One may then take the solution given by the Moore-Penrose pseudoinverse [13], for example. The condition $\operatorname{rank}\left(k_{0}\right)=m$ is non-restrictive. To see this, consider an LQR problem equivalent to (18). Then $k_{0}=-\left(R+B^{T} P_{0} B\right)^{-1} B^{T} P_{0} A_{0}$. Since usually the associated matrices $P_{0}, A_{0}$ and $R+B^{T} P_{0} B$ have full rank not less than $m$, it follows that it is sufficient with $\operatorname{rank}(B)=m$, which in general holds if there are no redundant inputs.

Theorem 4 implies local asymptotic stability of the closed loop. In many cases it is of more interest to investigate nonlocal asymptotic stability and also quantify the degree of suboptimality. These topics are closely interrelated and essentially depend on the cost function error that results from replacing $F^{T} x=F_{0}^{T} \zeta+F_{1}^{T} \varrho$ with $F_{0}^{T} \zeta$. The following result shows that the sub-optimality indeed depends critically on $F_{1}$. Before we state the result, we define the optimal cost associated with $J_{0}$ :

$$
V_{0}^{*}(x)=J_{0}\left(U_{0}^{*}(T x, K x), T x, K x\right)
$$

and the sub-optimal cost

$$
\hat{V}(x)=J\left(U_{0}^{*}(T x, K x), x\right)
$$

Lemma 2 If $F_{1}=0$ then $K=0$ is LQ optimal, and $\hat{V}(x)=$ $V^{*}(x)$ for all $x \in X$.

Proof. Directly from the definitions of the projections and the assumption $F_{1}=0$ we have

$$
\begin{aligned}
H^{-1} F^{T} & =H^{-1}\left(F_{0}^{T} V_{0}^{T}+F_{1}^{T} V_{1}^{T}\right) \\
& =H^{-1} F_{0}^{T} V_{0}^{T}=K_{0} T
\end{aligned}
$$

Observing that the first $m$ rows of the matrix on the left hand side equals $k_{L Q}$ and the first $m$ rows of the matrix on the right hand side equals $k_{0} T$, it follows from (23) that $k_{0} K=0$ and consequently that $K=0$ is LQ optimal. Then $\zeta^{*}=0$ and 
since $F_{0}^{T} \zeta=F^{T} x$ the value of the cost $J_{0}$ defined in (18) equals the value of the original cost $J$. Hence, the solution of the projected problem (18) - (19) equals the solution of the original problem (5)-(6), and their value functions must be equal.

This result is however of little practical use, since $F_{1} \neq 0$ because $F$ has full rank, in general, so we provide results that gives some further and a quantitative bound on the suboptimality.

Lemma 3 If $\zeta^{*}=K x$ where $K$ satisfies (23), then for all $U$ and $x \in X$ :

$$
J(U, \zeta, \varrho)=J_{0}\left(U, \zeta, \zeta^{*}\right)+x^{T}\left(V_{1} F_{1}+K^{T} F_{0}\right) U
$$

Proof. Using $Y=F H^{-1} F^{T}$, [4], it is straightforward to show that the last four terms in (17) add up to zero. Hence, we only need to consider the following terms

$$
\left(\zeta^{* T} F_{0}+\varrho^{T} F_{1}\right) U=x^{T}\left(K^{T} F_{0}+V_{1} F_{1}\right) U
$$

and the result is proven.

Theorem 5 If $\zeta^{*}=K x$ where $K$ satisfies (23), then for all $x \in X$ :

$$
0 \leq \hat{V}(x)-V^{*}(x) \leq \Delta(x)
$$

with $\Delta(x)=x^{T}\left(V_{1} F_{1}+K^{T} F_{0}\right)\left(U_{0}^{*}(T x, K x)-U^{*}(x)\right)$.

Proof. From Lemma 3

$$
\begin{aligned}
\hat{V}(x)= & J_{0}\left(U_{0}^{*}(T x, K x), T x, K x\right) \\
& \quad+x^{T}\left(V_{1} F_{1}+K^{T} F_{0}\right) U_{0}^{*}(T x, K x) \\
= & V_{0}^{*}(x)+x^{T}\left(V_{1} F_{1}+K^{T} F_{0}\right) U_{0}^{*}(T x, K x)
\end{aligned}
$$

Lemma 3 also gives

$$
\begin{aligned}
V_{0}^{*}(x)= & \min _{U x}\left(J\left(U, T x, V_{1}^{T} x\right)-x^{T}\left(V_{1} F_{1}+K^{T} F_{0}\right) U\right) \\
& \text { subject to } G U \leq W+E_{0} T x
\end{aligned}
$$

Due to feasibility of $U^{*}(x)$ the following inequality follows from sub-optimality

$$
V_{0}^{*}(x) \leq V^{*}(x)-x^{T}\left(V_{1} F_{1}+K^{T} F_{0}\right) U^{*}(x)
$$

Combining (34) and (35) gives (32).

Simulation example. A laboratory model helicopter (Quanser 3-DOF Helicopter) is sampled with interval $T=$ $0.01 s$, and the following state-space representation is obtained

$$
\begin{aligned}
A= & \left(\begin{array}{cccccc}
1 & 0 & 0.01 & 0 & 0 & 0 \\
0 & 1 & 0 & 0.01 & 0 & 0 \\
0 & 0 & 1 & 0 & 0 & 0 \\
0 & 0 & 0 & 1 & 0 & 0 \\
0.01 & 0 & 0 & 0 & 1 & 0 \\
0 & 0.01 & 0 & 0 & 0 & 1
\end{array}\right) \\
B= & \left(\begin{array}{cc}
0.0000 & 0.0000 \\
0.0001 & -0.0001 \\
0.0019 & 0.0019 \\
0.0132 & -0.0132 \\
0 & 0 \\
0 & 0
\end{array}\right)
\end{aligned}
$$

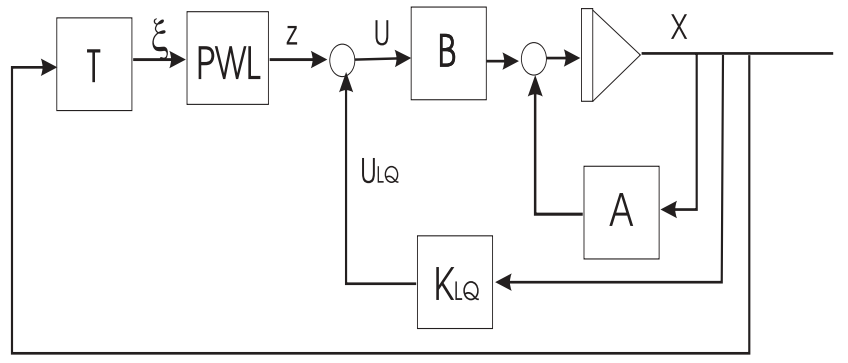

Figure 3: Feedback structure II.

The states of the system are $x_{1}$ - elevation, $x_{2}$ - pitch angle, $x_{3}$ - elevation rate, $x_{4}$ - pitch angle rate, $x_{5}$ - integral of elevation error, and $x_{6}$ - integral of pitch angle error. The inputs to the system are $u_{1}$ - front rotor voltage and $u_{2}$ - rear rotor voltage. Assume the state is to be regulated to the origin with the following constraints on the inputs and pitch and elevation rates $-1 \leq u_{1} \leq 3,-1 \leq u_{2} \leq 3,-0.44 \leq y_{1} \leq 0.44$, and $-0.6 \leq y_{2} \leq 0 . \overline{6}$ with

$$
C=\left(\begin{array}{llllll}
0 & 0 & 1 & 0 & 0 & 0 \\
0 & 0 & 0 & 1 & 0 & 0
\end{array}\right)
$$

The LQ cost function is given by $Q=$ $\operatorname{diag}(100,100,10,10,400,160)$, and $R=I_{2 \times 2}$. We assume the horizon $N=50$ and the input trajectory is a piecewise constant function of time parameterized by 3 parameters per input as in [10]. For this 6th order system the observability matrix of the system $(A, C)$ has rank 2 , since there is one integrator between the inputs and each of the two constrained states. Hence, $p=m=2$ and the dimension of the mp-QP parameter space is reduced from 6 to 4 . This leads to a reduction in the number of regions in the partition generated by the mp-QP algorithm [6] from 4279 to 1253 . The simulation in Figure 2 starting from the initial state $x(0)=(0.65,0.65,0,0,-0.65,-0.45)$ shows that the resulting control system does not violate the constraints and that the performance degradation is not prohibitive. A Monte Carlo simulation over 469 random initial conditions that give feasible trajectories in the set $X=[-0.75,0.75]^{4} \times[-1,1]^{2}$ shows that with the sub-optimal control the cost is increased by $8.7 \%$ on average and $146.3 \%$ in the worst case.

\section{Feedback structure II}

Consider the feedback structure II shown in Figure 3. It contains an inner linear feedback that is LQ-optimal for the unconstrained system, and a PWL outer feedback defined on a lower dimensional sub-space of the state space. The outer loop will be designed by solving an mp-QP to modify the LQ feedback (in a possibly sub-optimal way) such that the constraint are fulfilled only to some tolerance.

Using arguments similar to Theorem 3, the number of nonzero singular values of $S$ equals the rank of the observability matrix of the system $\left(A-B k_{L Q}, C\right)$, at least for $N \geq n$. We notice that in this case any structural properties of the system $(A, B, C)$ will typically be lost due to the LQ feedback. Hence, only in trivial special cases will the observability matrix of the system $\left(A-B k_{L Q}, C\right)$ not have full rank. However, we may still exploit similar ideas to derive a reduced-order mp-QP if some violation of the constraints are allowed. This is easily achieved by defining a threshold on the singular values of $S$ such that the constraints (8) are equivalently represented as

$$
G z \leq W+S_{0} \xi+\varepsilon
$$



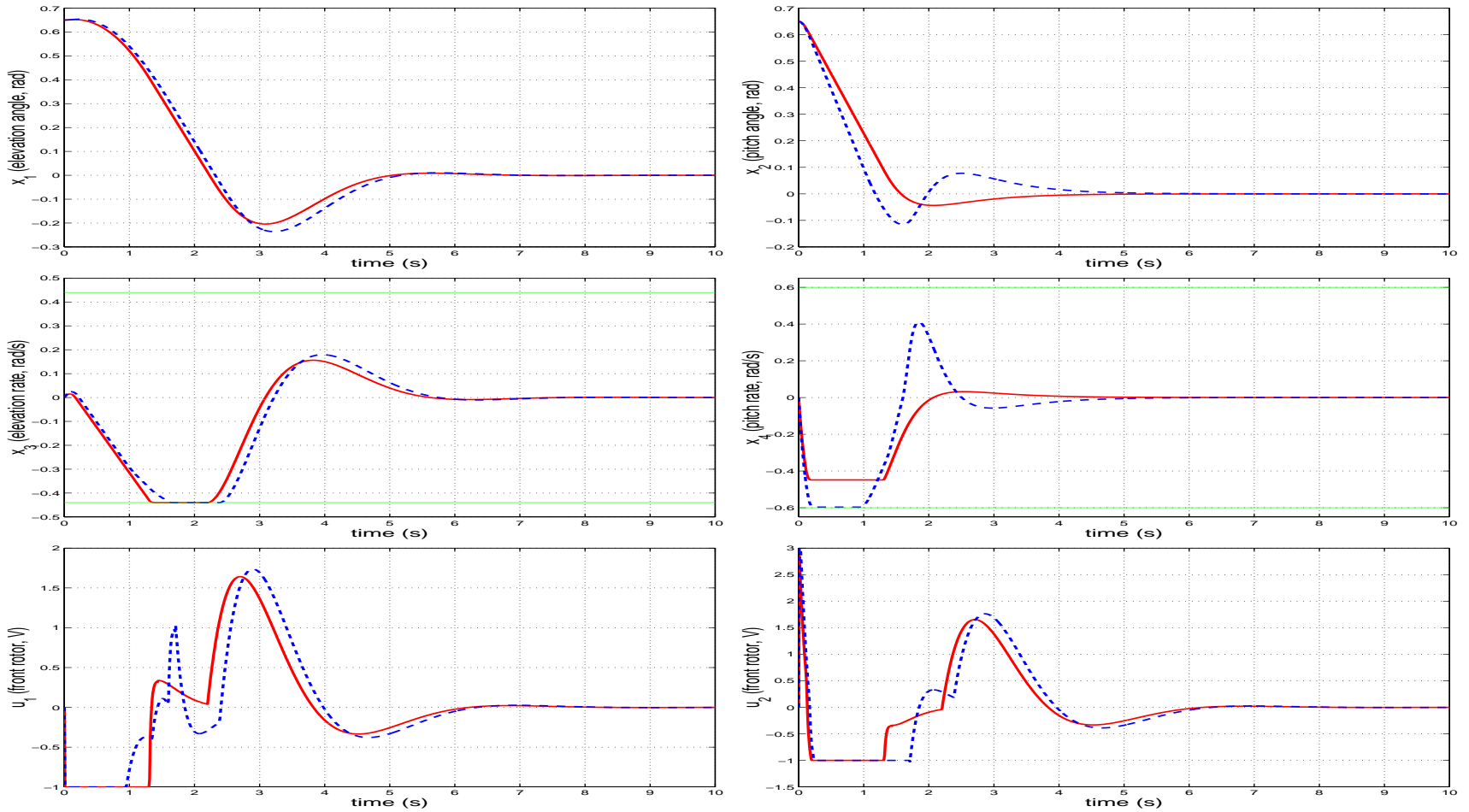

Figure 2: Simulation results: Exact explicit LQR (solid curves), and approximate (dashed-dotted curves).

with $\xi=T_{0} x, T_{0}=V_{0}^{T}, S_{0}=U_{0} \Sigma_{0}$, where $U_{0}, V_{0}, \Sigma_{0}$ are the sub-matrices of the singular value decomposition $S=U \Sigma V^{T}$ corresponding to singular values larger than a given threshold $\sigma_{0} \geq 0$. Likewise, $\varepsilon=U_{1}^{T} \Sigma_{1} V_{1}^{T}$, where $U_{1}, V_{1}, \Sigma_{1}$ are the sub-matrices of the singular value decomposition corresponding to singular values that are not larger than $\sigma_{0}$. In general $\operatorname{dim}(\xi) \leq \operatorname{dim}(x)$, and a uniform a priori bound on $\varepsilon$ follows directly from properties of the singular value decomposition [13]:

Lemma 4 Let $\sigma_{t}$ be the largest singular value of $S$ that satisfies $\sigma_{t} \leq \sigma_{0}$, and assume $X$ is a compact polyhedral set. Then $\max _{x \in X}\|\varepsilon\|_{2} \leq \sigma_{t} \max _{x \in X}\|x\|_{2}$.

It can be observed that the slack variable $\varepsilon$ in (36) will be uniformly small if the threshold $\sigma_{0}$ is small. Then $\varepsilon$ may be neglected, which suggests the following reduced-dimension mp-QP, defined on the projection of $X$ onto the sub-space $\Xi=\left\{\xi \mid \xi=T_{0} x, x \in X\right\}$ spanned by the rows of $T_{0}$ :

$$
\begin{aligned}
V_{z, 0}^{*}(\xi) & =\min _{z} \frac{1}{2} z^{T} H z \\
\text { subject to } & G z \leq W+S_{0} \xi
\end{aligned}
$$

Since the constraints are relaxed by an amount $\varepsilon$, the optimal solution may violate the constraints.

The control is chosen according to the receding horizon policy

$$
u^{*}(t)=u_{L Q}(t)+z_{0,0}^{*}(\xi(t))
$$

where $z_{0,0}^{*}$ denotes the $m$ first components of the vector $z_{0}^{*}$ that solves (37)-(38). As usual, when using the singular value decomposition, appropriate scaling is important. Essentially, the inequalities should be scaled according to some prioritization of the constraints. In other words, constraints where only small violations are tolerated should be scaled up, while constraints which tolerate large violations should be scaled down.

It may be a requirement that certain constraints are not allowed to be violated. This is usually the case for input constraints, which are often physical limitations rather than operational constraints. In order to fulfill hard input constraints with the receding horizon control (39), information about $u_{L Q}(t)$ is sufficient:

Lemma 5 If $\operatorname{span}\left(k_{L Q}\right) \subseteq \operatorname{span}\left(T_{0}\right)$, then $S_{0}$ in (38) can be chosen such that the input constraints $u_{\min } \leq u(t) \leq u_{\max }$ are satisfied at the optimum for any $x(t) \in X$.

Proof. Let the sub-matrices $\tilde{G}, \tilde{W}$ and $\tilde{S}$ correspond to the constraints $u_{\min } \leq u(t) \leq u_{\max }$ in the form

$$
\tilde{G} z^{*}(t) \leq \tilde{W}+\tilde{S} x(t)
$$

It is straightforward to see that

$$
\begin{aligned}
& \tilde{G}=\left(\begin{array}{cc}
I_{m \times m} & 0_{m \times m(N-1)} \\
-I_{m \times m} & 0_{m \times m(N-1)}
\end{array}\right), \\
& \tilde{W}=\left(\begin{array}{c}
u_{\max } \\
-u_{\min }
\end{array}\right), \tilde{S}=\left(\begin{array}{c}
k_{L Q} \\
-k_{L Q}
\end{array}\right)
\end{aligned}
$$

since $S=E+K_{L Q}$ and $E=0$ for input constraints. Now consider the corresponding sub-matrices of the reduced constraints (38), i.e.

$$
\tilde{G} z(t) \leq \tilde{W}+\tilde{S}_{0} \xi(t)=\tilde{W}+\tilde{S}_{0} T_{0} x(t)
$$


The result follows since the reduced and original constraints can be made equivalent by the choice $\tilde{S}_{0}^{T}=\left(\mathcal{X}^{T},-\mathcal{X}^{T}\right)$, swhere $\mathcal{X} \in \mathbb{R}^{m \times p}$ is a matrix such that $\mathcal{X} T_{0}=k_{L Q}$. This matrix must exist and be of rank $m \operatorname{since} \operatorname{span}\left(k_{L Q}\right) \subseteq$ $\operatorname{span}\left(T_{0}\right)$.

According to Lemma 5 the rows of the projection $T_{0}$ should include the normalized rows of $k_{L Q}$. In order to minimize violation of the state constraints, we suggest the following procedure to choose the reduced constraints that also includes the most significant directions of the sub-space orthogonal to the sub-space spanned by $k_{L Q}$. Let the rows of the matrix $k_{L Q}^{\perp}$ contain a basis for null $\left(k_{L Q}\right)$. Assuming without loss of generality that $k_{L Q}$ has row rank $m$ such that its null space basis $k_{L Q}^{\perp}$ has rank $n-m$, we define $D=S\left(\begin{array}{c}k_{L Q} \\ k_{L Q}^{\perp}\end{array}\right)^{-1}$. Hence, $S=D_{1} k_{L Q}+D_{2} k_{L Q}^{\perp}$ where $D_{1}$ contains the $m$ first columns of $D$, and $D_{2}$ the remaining $n-m$ columns. Consider the singular value decomposition $D_{2} k_{L Q}^{\perp}=U \Sigma V^{T}$ which gives $S=\mathcal{S}_{0} \xi+\varepsilon$, where $\mathcal{S}_{0}=\left(D_{1}, U_{0} \Sigma_{0}\right)$, $\mathcal{T}_{0}=\left(\begin{array}{c}k_{L Q} \\ V_{0}^{T}\end{array}\right)$, and $\varepsilon=U_{1} \Sigma_{1} V_{1}^{T} x$ where $U_{0}, \Sigma_{0}, V_{0}$ and $U_{1}, \Sigma_{1}, V_{1}$ are as defined above. This leads to the following mp-QP

$$
\begin{aligned}
\mathcal{V}_{z, 0}^{*}(\xi)= & \min _{z} \frac{1}{2} z^{T} H z \\
\text { subject to } & G z \leq W+\mathcal{S}_{0} \beta
\end{aligned}
$$

with $\beta=\mathcal{T}_{0} x$.

Example, continued. With the above LQ design the $S$ matrix has the following singular values: $64.0025,32.2419$, 5.6246, 2.8686, 1.2025, and 1.0842. Assume we neglect the two smallest singular values, which yields an approximate mp-QP defined on a 4-dimensional parameter space. The PWL feedback laws with exact and approximate explicit MPC with and without hard input constraints have 4279, 1930 and 1936 regions, respectively. Hence, there is significant complexity reduction. Table 1 summarizes the performance degradation and constraint violations over 461 random initial states. Since the constraint are allowed to be violated the average change in cost is close to zero, so we report instead both the maximum increase and decrease in cost relative to the optimal cost. We notice that the ratio between the largest and smallest singular values is small, such that significant constraint violations and performance degradation is expected in this example.

\begin{tabular}{lcc}
\hline & $\begin{array}{c}\text { Soft input } \\
\text { constraints }\end{array}$ & $\begin{array}{c}\text { Hard input } \\
\text { constraints }\end{array}$ \\
\hline Max. violation $y_{1}$ & 0.13 & 0.14 \\
Max. violation $y_{2}$ & 0.06 & 0.07 \\
Max. violation $u_{1}$ & 0.13 & - \\
Max. violation $u_{2}$ & 0.12 & - \\
Max. increase cost & $81.8 \%$ & $64.4 \%$ \\
Max. decrease cost & $34.2 \%$ & $36.7 \%$ \\
\hline
\end{tabular}

Table 1: Summary of Monte Carlo simulations.

\section{Conclusions}

Methods for reducing the dimension of the parameter space of mp-QP solutions to explicit constrained LQR problems are investigated. It is shown that for systems with certain structures such dimension reduction can be achieved by state space projections that leads to $\mathrm{mp}-\mathrm{QPs}$ that require less offline and online computations and computer memory. Examples indicate that the performance degradation may be acceptable in some applications.

\section{References}

[1] D. Chmielewski and V. Manousiouthakis, "On constrained infinite-time linear quadratic optimal control," Systems and Control Letters, vol. 29, pp. 121-129, 1996.

[2] P. O. M. Scokaert and J. B. Rawlings, "Constrained linear quadratic regulation," IEEE Trans. Automatic Control, vol. 43, pp. 1163-1169, 1998.

[3] A. Bemporad, M. Morari, V. Dua, and E. N. Pistikopoulos, "The explicit solution of model predictive control via multiparametric quadratic programming," in Proc. American Control Conference, Chicago, 2000, pp. 872-876.

[4] A. Bemporad, M. Morari, V. Dua, and E. N. Pistikopoulos, "The explicit linear quadratic regulator for constrained systems," Automatica, vol. 38, pp. 3-20, 2002.

[5] M. Seron, J. A. De Dona, and G. C. Goodwin, "Global analytical model predictive control with input constraints," in Proc. IEEE Conf. Decision and Control, Sydney, 2000, pp. TuA05-2.

[6] P. Tøndel, T. A. Johansen, and A. Bemporad, "An algorithm for multi-parametric quadratic programming and explicit MPC solutions," in Proc. IEEE Conf. Decision and Control, Orlando, 2001, pp. TuP11-4.

[7] A. Bemporad and C. Filippi, "Suboptimal explicit MPC via approximate quadratic programming," in Proc. IEEE Conf. Decision and Control, Orlando, 2001, pp. FrP08-5.

[8] T. A. Johansen and A. Grancharova, "Approximate explicit model predictive control implemented via orthogonal search tree partitioning," in Preprints, IFAC World Congress, Barcelona, 2002, http://www.itk.ntnu.no/ansatte/Johansen_Tor.Arne/mpqptree-ifac.pdf.

[9] F. Borrelli, M. Baotic, A. Bemporad, and M. Morari, "Efficient on-line computation of explicit model predictive control," in Proc. IEEE Conf. Decision and Control, Orlando, 2001, pp. TuP11-2.

[10] P. Tøndel and T. A. Johansen, "Complexity reduction in explicit model predictive control," in Preprints, IFAC World Congress, Barcelona, 2002, http://www.itk.ntnu.no/ansatte/Johansen_Tor.Arne/mpqpifac-2002.pdf.

[11] T. A. Johansen, I. Petersen, and O. Slupphaug, "On explicit suboptimal LQR with state and input constraints," in Proc. IEEE Conf. Decision and Control, Sydney, 2000, pp. TuM05-6.

[12] T. A. Johansen, I. Petersen, and O. Slupphaug, "Explicit suboptimal linear quadratic regulation with input and state constraints," Automatica, vol. 38, pp. 1099-1112, July 2002.

[13] G. H. Golub and C. F. van Loan, Matrix Computations, The Johns Hopkins University Press, Baltimore, MD, 1989.

[14] T. Kailath, Linear Systems, Prentice Hall, Englewood Cliffs, N.J., 1980. 